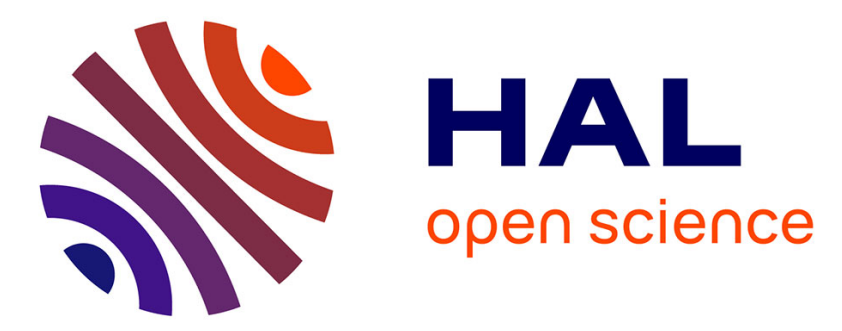

\title{
Low-Cost EVM Measurement of ZigBee Transmitters From 1-bit Undersampled Acquisition
}

Thibault Vayssade, Florence Azaïs, Laurent Latorre, François Lefèvre

\section{To cite this version:}

Thibault Vayssade, Florence Azaïs, Laurent Latorre, François Lefèvre. Low-Cost EVM Measurement of ZigBee Transmitters From 1-bit Undersampled Acquisition. IEEE Transactions on Computer-Aided Design of Integrated Circuits and Systems, 2021, 40 (11), pp.2400-2410. 10.1109/TCAD.2020.3043318 . lirmm-03426162

\section{HAL Id: lirmm-03426162 \\ https://hal-lirmm.ccsd.cnrs.fr/lirmm-03426162}

Submitted on 12 Nov 2021

HAL is a multi-disciplinary open access archive for the deposit and dissemination of scientific research documents, whether they are published or not. The documents may come from teaching and research institutions in France or abroad, or from public or private research centers.
L'archive ouverte pluridisciplinaire HAL, est destinée au dépôt et à la diffusion de documents scientifiques de niveau recherche, publiés ou non, émanant des établissements d'enseignement et de recherche français ou étrangers, des laboratoires publics ou privés. 


\title{
Low-cost EVM measurement of ZigBee transmitters from 1-bit under-sampled acquisition
}

\author{
T. Vayssade, Member, IEEE, F. Azaïs, Member, IEEE, L. Latorre, Member, IEEE, and F. Lefèvre,
}

\begin{abstract}
Error Vector Magnitude (EVM) or alternately Offset Error Vector Magnitude (OEVM) is one of the most important performance to verify for RF circuits such as ZigBee transmitters in order to ensure that the quality of the generated modulated-signal complies with the requirements of the wireless communication standard. The conventional solution to measure this performance relies on the use of an Automatic Test Equipment (ATE) equipped with expensive RF channels. This paper presents a low-cost solution that permits to realize EVM measurement using only a standard digital ATE. The approach is based on 1-bit under-sampled acquisition of the RF modulatedsignal by a digital tester channel associated with a specificallytailored processing algorithm. This algorithm involves many different steps that are detailed in this paper, including phase and amplitude fluctuation extraction, RF signal reconstruction, symbol clock recovery, symbol bits detection and synthesis of reference data for EVM calculation. The proposed digital test solution is first evaluated through lab hardware experiments and then validated with EVM measurements on an industrial ATE.
\end{abstract}

Index Terms - RF test, digital ATE, EVM measurement, digital signal processing, wireless communication, ZigBee, OQPSK

\section{INTRODUCTION}

$\mathrm{W}$ ITH a forecast of 38.9 billion of connected devices in the world in 2025 [1] the market for IOT-dedicated integrated circuits is getting very competitive. Typical examples of such circuits are Systems on Chip (SoC) that integrate the application processor together with embedded RF front-end. Along with the growth of industrial and home appliances, protocols such as BLE and Zigbee ${ }^{\circledR}$ have become ubiquitous for short range communicating devices.

The cost of manufacturing test has long been a major concern for integrated devices, both digital and analog. In particular, analog and RF testing is expensive because it implies the direct characterization of key specifications, involving time and high-priced measurement resources [2]. The idea in this work is to replace the costly RF acquisition hardware so-far involved in manufacturing testing with a simple 1-bit synchronous digitizing process. The envisioned

This work has been carried out under the framework of PENTA-EUREKA project "HADES: Hierarchy-Aware and secure embedded test infrastructure for Dependability and performance Enhancement of integrated Systems".

T. Vayssade, F. Azaïs, L Latorre are with University of Montpellier, CNRS, LIRMM, 34095 Montpellier, France (e-mail: author@ lirmm.fr)

F. Lefèvre is with NXP Semiconductors, 14000 Caen, France, (e-mail: francois.lefevre@nxp.com)
ADC is basically the clocked comparator stage found in standard digital input channel of Automatic Test Equipment (ATE). That resource is much less expensive than RF instrumentation, and the available channel count is usually large enough to implement parallel testing of several devices (multi-site testing), therefore reducing the average test time per device.

The idea of using digital resources for RF testing is not new. In [3], a solution based on an additional reference transceiver and an FPGA at board level was proposed to interface with the digital domain of the ATE. In [4], a dedicated self-test processor is embedded into the SoC under test to provide low-frequency digital output. An off-chip direct modulation / demodulation scheme was proposed in [5]. All the above involve additional hardware resource, either at silicon or board level. Direct 1-bit acquisition has also been explored for analog signals, but at lower frequencies, for instance in [6] for amplitude and offset measurement of triangular waves, or in [7] for demodulation of basic AM/FM schemes. Regarding RF signals, previous work on phase noise characterization in tuner devices validated the concept of direct 1-bit sampling [8].

In this work, we focus on Offset Error Vector Magnitude (OEVM) measurement on $2.4 \mathrm{GHz}$ Zigbee ${ }^{\circledR}$ signals, which is one of the key specifications that must be verified. It is challenging because of the complex modulation scheme of Zigbee ${ }^{\circledR}$ transceivers. In addition, actual products exhibit a very low OEVM level (below 1\%), thus requiring precise knowledge of signal characteristics to obtain meaningful results.

This paper is organized as follows. Section II gives some background about the characteristics of the signal under test and the current industrial test practice for EVM measurement. Section III introduces the principle of the proposed digital test strategy and Section IV details the practical implementation of the post-processing algorithm. Finally, before conclusion, hardware experimental results are presented in Section V.

\section{BACKGROUND}

\section{A. Signal Under Test Characteristics (ZigBee)}

The main characteristics of the signal to be analyzed, as specified by the IEEE Std $802.15 .4^{\mathrm{TM}}$, are briefly summarized in this section. In this work, we focus on the $2.4 \mathrm{GHz}$ band, which is an ISM band accepted worldwide.

In this band, the standard specifies that the modulation scheme is Offset Quadrature Phase Shift Keying (OQPSK) 
with half-sine pulse shaping. It is a form of phase-shift keying which uses four angles separated by $90^{\circ}$ spacing corresponding to the symbol bits 00, 01, 10 and 11 respectively. The generic block diagram of an OQPSK modulator with half-sine pulse shaping is given in Fig.1. As in a conventional QPSK modulator, the input data stream is first split in two branches and bipolar NRZ encoding is applied on both even bits fed in the $I$ branch and odd bits fed in the $Q$ branch. However, prior to the multiplication of these components with the orthogonal sine and cosine functions, half-sine pulse shaping is applied on both the signals in the $I$ and $Q$ branches and an offset of one-bit period (half bitsymbol period) is added on the signal in the $Q$ branch. Finally, the two signals are added to form the modulated signal.

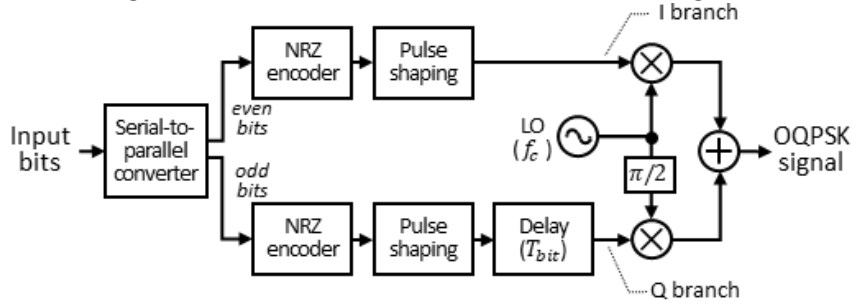

Fig. 1. Block diagram of OQPSK modulator with half-sine pulse shaping.

Compared to a conventional QPSK modulation scheme, the offset of one-bit period in the $Q$ branch permits to improve the spectral efficiency. Indeed, thanks to this additional delay, signals in the $I$ and $Q$ branches will never change at the same time, limiting the maximum phase jump to $90^{\circ}$ instead of $180^{\circ}$. The use of half-sine pulse shaping also contributes to an enhanced spectral efficiency. It smooths the phase transitions (continuous phase travelling along the trigonometric circle) and therefore ensures a constant modulation envelope.

Finally, the last feature specified by the standard is the use of Direct Sequence Spread Spectrum (DSSS) technique. The general principle consists in multiplying the data being transmitted by a noise signal in order to reduce overall interference. More precisely with DSSS, the message bits are modulated by a pseudorandom bit sequence called spreading sequence, each bit of the spreading sequence (called chip) having a much shorter duration than the original message bits.

TABLE I

FREQUENCY BAND, MODULATION FORMAT AND DATA RATES

\begin{tabular}{|l|l|l|l|l|}
\hline \hline $\begin{array}{l}\text { Frequency } \\
\text { Band (MHz) }\end{array}$ & $\begin{array}{l}\text { Spreading } \\
\text { Technique }\end{array}$ & $\begin{array}{l}\text { Modulation } \\
\text { Format }\end{array}$ & $\begin{array}{l}\text { Input } \\
\text { Data Rate }\end{array}$ & $\begin{array}{l}\text { Chip } \\
\text { Rate }\end{array}$ \\
\hline $2400-2483.5$ & $\begin{array}{l}\text { 16-ary } \\
\text { orthogonal DSSS }\end{array}$ & $\begin{array}{l}\text { OQPSK with half- } \\
\text { sine pulse shaping }\end{array}$ & $250 \mathrm{~kb} / \mathrm{s}$ & $2 \mathrm{Mchip} / \mathrm{s}$ \\
\hline \hline
\end{tabular}

Practically, the standard specifies the utilization of a 16-ary quasi-orthogonal DSSS modulation technique, in which every four bits of the input message are mapped into one of the 16 Pseudo-random Noise (PN) sequence composed of 32 chips. The PN sequences are concatenated and the aggregate chip sequence is then modulated onto the carrier using OQPSK. The main characteristics of the signal in terms of frequency band, modulation format and data rates are summarized in Table I. With an input data rate of $250 \mathrm{~kb} / \mathrm{s}$, the chip rate at the input of the OQPSK modulator is $2 \mathrm{Mchip} / \mathrm{s}(1 \mathrm{Mb} / \mathrm{s}$ in the $I / Q$ branches). It is worth noting that although the physical generated signal is at $2.4 \mathrm{GHz}$, the useful bit information transmitted by the circuit is only at $2 \mathrm{MHz}$. We intend to exploit this attribute for the development of our digital test solution.

\section{B. Current test practice}

The current industrial practice for production testing of RF devices relies on the use of an ATE equipped with RF tester channels. As illustrated in Fig.2, such channels comprise specific hardware resources (mixer, local oscillator, filters, analog-to-digital converter) dedicated to the acquisition of an RF signal. More precisely, the RF signal is first downconverted towards the Intermediate Frequency (IF) band by mixing it with a local oscillator. The resulting analog signal is then filtered. Finally, it is converted into the digital domain using an ADC and the digitized version of the down-converted RF signal is stored in the ATE memory.

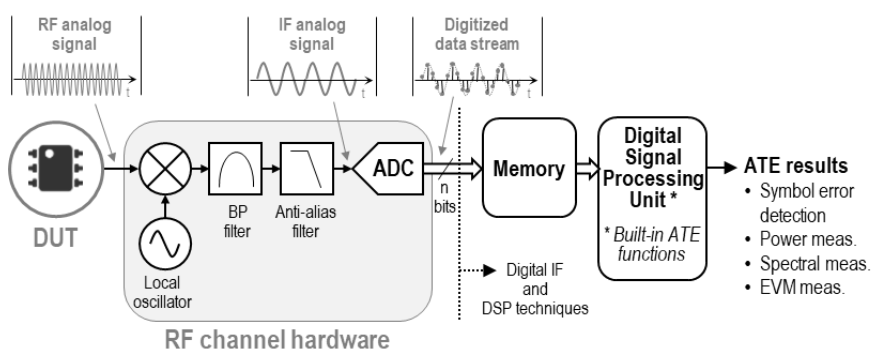

Fig. 2. Current industrial setup for testing ZigBee devices.

From there, software DSP procedures using built-in ATE functions are applied to compute various features of the signal under test. In particular, code-domain sequences can be extracted in order to implement symbol error detection and verify the basic functionality of the device. Performance measurements can also be realized, i.e. power, spectral and EVM measurements, in order to verify that the device performances comply with the product specifications. Although this conventional approach enables the full verification of an RF device, its main drawback is its cost. Indeed, because of the required hardware resources with highlevel performances, the cost of an RF tester channel is extremely high and constitutes a major contributor to the RF testing cost.

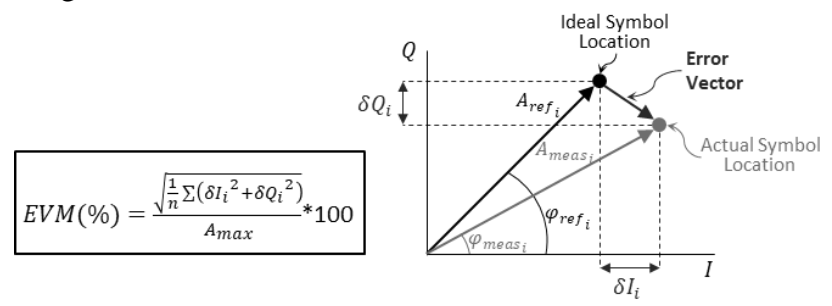

Fig. 3. Error Vector Magnitude (EVM).

In this paper, we focus on EVM measurements, which is the most widely used metric to express the modulation quality in wireless communication systems. Basically, it is a measure of the signal constellation deviation from its ideal reference. It is computed from error vectors defined in the I-Q plane at the instants in time when symbols are detected. As illustrated in Fig.3, the error vector is the difference at a given time between the actual symbol location and the ideal one. EVM is then 
defined as the Root Mean Square (RMS) amplitude of the error vector over a sequence of emitted symbols, normalized to ideal signal amplitude reference (maximum signal amplitude in case of ZigBee as specified by IEEE Std 802.15.4TM).

The ATE manufacturer provides a specific software built-in function to realize such EVM measurements on the digitized data obtained from an RF acquisition.

IEEE Std $802.15 .4^{\mathrm{TM}}$ specifies a pass limit of $35 \%$ for the EVM. Yet, in case of OQPSK modulation scheme, due to the one-bit period offset between I and Q channels, an Offset Error Vector Magnitude (OEVM) is calculated instead of the EVM. As a rule of thumb, it is common to consider that OEVM is about 5 times smaller than EVM for the same modulated signal. That would lead to a pass limit of $6 \%$ for the OEVM. In practice, manufacturers set this limit much below, around 1\%. OEVM being a particular form of the generic EVM, both names are used all along the paper.

\section{PROPOSED DigITAL TEST SOLUTION}

Our strategy towards the reduction of RF testing cost is to develop new solutions allowing the replacement of expensive RF tester channels by low-cost standard digital tester channels. The idea is to perform 1-bit acquisition of the RF signal using a standard digital tester channel, and then to develop dedicated post-processing algorithms able to evaluate the RF signal characteristics from the binary vector stored in the ATE memory. This approach is illustrated in Fig.4.

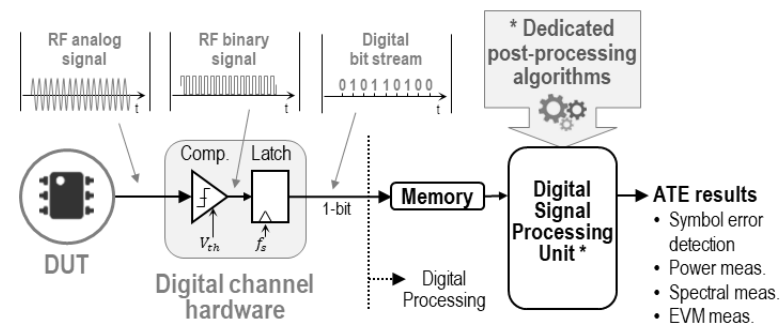

Fig. 4. Proposed digital approach for testing ZigBee devices.

The fundamental concept behind this approach is to exploit the properties of the hardware resources comprised in a digital tester channel, i.e. a comparator and a latch. These resources implement a level-crossing operation followed by sampling on the signal passing through them, which means that they transform the amplitude, frequency and/or phase information contained in the analog signal into a timing information contained in the resulting bitstream. The objective is to extract this information and process it in order to retrieve the desired RF signal characteristics. Key challenges are (i) to determine appropriate conditions for the digital capture that permit to preserve the essential information contained in the RF signal and (ii) to define the proper dedicated processing algorithms that will run on the Digital Signal Processing unit of the ATE.

Regarding the digital capture, some works have already exploited the principle of 1-bit acquisition, but based on an oversampled acquisition [6-8]. In this work, we target an RF modulated-signal with a carrier at $2.4 \mathrm{GHz}$, which means that oversampling cannot be used since it exceeds the capability of a standard digital ATE channel (e.g. maximum sampling rate of $1.6 \mathrm{Gbps}$ for PinScale ${ }^{\circledR} 1600$ of Advantest V93k ATE). The proposed solution therefore relies on an under-sampled 1bit acquisition. More precisely, the idea is to under-sample the RF binary signal generated by level-crossing operation with a sampling rate $f_{s}$ close to half the carrier frequency $f_{c}$ :

$$
f_{s}=f_{c} / 2 \pm f_{d}
$$

where $f_{d}$ is the frequency deviation between the sampling frequency and half the carrier frequency.

This approach is a particular case of what is known in the literature as harmonic sampling. This principle is notably used in instrumentation dedicated to the analysis of high frequency signals such as large-signal network analyzers $[9,10]$. Essentially, the idea is to shift the signal to be measured from a high frequency band to baseband, by taking advantage of the aliasing effect. The technique is particularly adapted to the analysis of narrow-bandwidth signals, which corresponds to our context of ZigBee signals. The main requirement is that the sampling frequency should be set at least at twice the bandwidth of the signal to be analyzed $\left(f_{s}>2 B\right)$ in order to avoid overlap between aliases in the baseband. A more general criterion to implement this under-sampling scheme is given by:

$$
\begin{aligned}
& \frac{2 f_{c}+B}{k} \leq f_{s} \leq \frac{2 f_{c}-B}{k-1} \\
& \text { for any integer } k \text { satisfying } 2<k \leq\left\lfloor\frac{1}{2}+\frac{f_{c}}{B}\right\rfloor
\end{aligned}
$$

where $B$ is the bandwidth of the signal to be analyzed.

In our case, we deal with a very narrow-bandwidth signal (spectral requirements specified by IEEE Std $802.15 .4^{\mathrm{TM}}$ are given at $\pm 3.5 \mathrm{MHz}$ from the carrier frequency) and we have 26 possible channels separated by $5 \mathrm{MHz}$ starting from $2.405 \mathrm{GHz}$ up to $2.48 \mathrm{GHz}$.

The sampling frequency may be tuned according the actual Zigbee ${ }^{\circledR}$ channel used during the test. Let us consider for instance the first channel with frequency spread over the $2.4 \mathrm{GHz}-2.41 \mathrm{GHz}$ range. Taking these constraints into account, $k=4$ is sufficient to comply with the maximum $1.6 \mathrm{Gbps}$ sampling rate of a standard digital channel. This justifies the choice of a sampling rate close to half the carrier frequency as expressed in (1), with a sampling frequency $f_{s}$ that can be freely selected between $1.205 \mathrm{GHz}$ and $1.6 \mathrm{GHz}$.

In this specific context, we have many possibilities for the choice of the sampling frequency value. In particular, the value of the frequency deviation $f_{d}$ can be freely chosen between $2.5 \mathrm{MHz}$ and $397.5 \mathrm{MHz}$. A central element to take into consideration is that the timing resolution obtained on the baseband digital signal is directly related to the frequency deviation, i.e. the smaller the frequency deviation, the higher the timing resolution in terms of available number of samples per period. This is particularly important in our context where we intend to analyze the time-domain characteristics of this signal in order to extract information on both amplitude and phase of the original RF signal. It is clear that the better the timing resolution of the baseband digital signal, the more accurate the extracted information will be. In this work, we choose a frequency deviation around $10 \mathrm{MHz}$, which offers an 
extra margin with respect to the non-overlapping criterion but still ensures a good resolution for the analysis of the baseband digital signal, i.e. around 60 samples for each signal period.

To summarize, the proposed 1-bit under-sampling process produces a digital signal with a fundamental beat frequency at $f_{b}=\left|2 f_{s}-f_{c}\right|=2\left|f_{d}\right|$ that still contains relevant information on the phase and amplitude of the original RF signal, provided an adequate sampling frequency choice. Regarding postprocessing algorithms, we have already developed solutions for symbol error detection [11] and power and spectral measurements [12]. In this paper, we complete the work by proposing an original algorithm dedicated to EVM measurements. With this new algorithm, all the tests specified by the IEEE Std 802.15.4 with respect to the transmitter chain can be implemented using a standard digital ATE.

A global overview of the post-processing algorithm dedicated to EVM measurement is given in Fig.5. It involves three main steps. The first one is dedicated to the processing of the binary discrete-time vector captured by the ATE to extract relevant information regarding amplitude and phase fluctuation of the RF signal and convert this information into analog continuous-time waveforms. This information is then used in the second step in two parallel branches dedicated to (i) reference data generation and (ii) measurement data preparation. Note there are interactions between these two branches, for instance regarding correction of the extracted phase fluctuation and determination of the sampling instants for both measured and reference data. Finally, the last step of the algorithm simply consists in the EVM calculation from measured and reference constellations. These different steps are detailed in the following section.

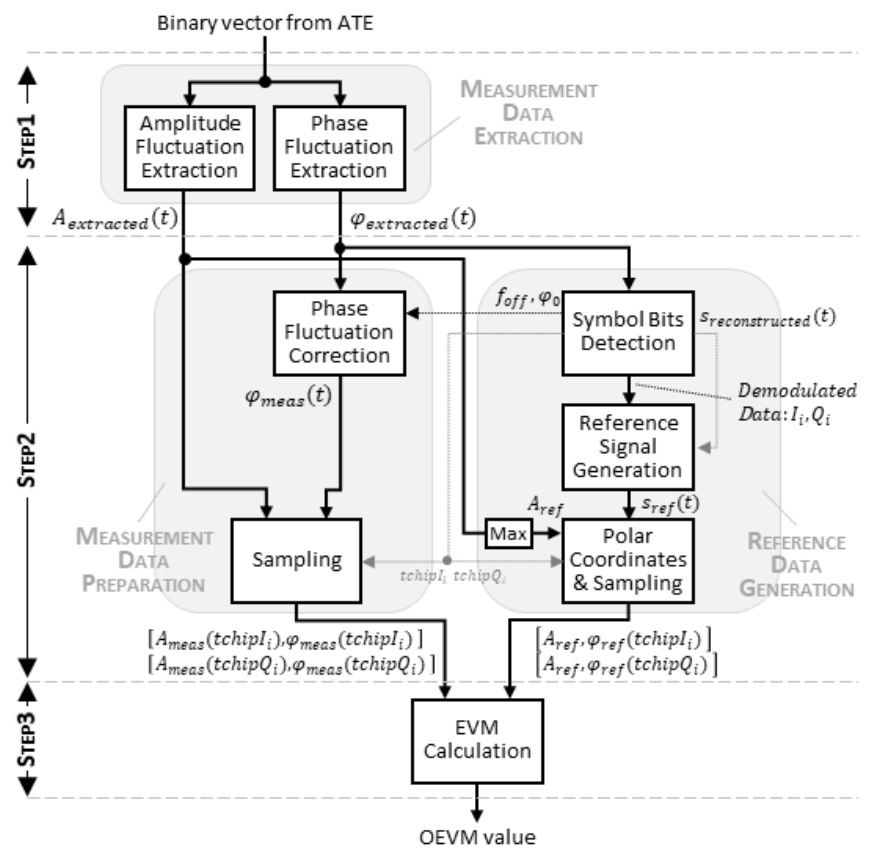

Fig. 5. Block diagram of the post-processing algorithm for EVM measurement.

\section{Post-Processing Algorithm}

\section{A. Step 1: Measurement data extraction}

The first step of the algorithm consists in processing the binary vector captured by the ATE in order to obtain analog continuous-time estimation of both the phase and amplitude fluctuations of the RF signal. These two estimations can be realized in parallel.

\section{1) Phase fluctuation extraction}

A first task of the algorithm is to retrieve the phase variation of the RF modulated signal. This is an essential task since all the information delivered by a ZigBee transmitter translates in phase variations of the RF modulated signal. The implemented solution relies on the work developed in [10] for symbol error detection using digital test resources. It involves several operations as depicted in Fig.6, which are briefly summarized hereafter.

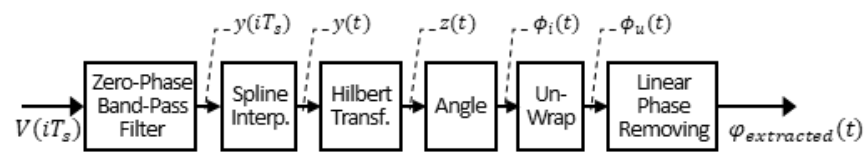

Fig. 6. Phase fluctuation extraction from binary vector captured by the ATE.

The digital signal $V\left(i T_{S}\right)$ captured by the ATE is first filtered by a zero-phase band-pass filter centered on the square-wave beat frequency in order to eliminate harmonics introduced by 1-bit quantization. Note that it is important to use a zero-phase filter to preclude from any additional delay in the signal. Although zero-phase filtering cannot be physically integrated for real-time signals, it can be easily implemented in the context of a post-processing procedure using forwardbackward filtering. The resulting signal is a discrete-time sinewave $y\left(i T_{s}\right)$. Spline interpolation followed by Hilbert Transform are then applied to obtain a time-continuous expression of the signal in the complex plane $z(t)$. The instantaneous phase $\Phi_{i}(t)$ is then computed by taking the angle of the complex signal $z(t)$ with $\Phi_{i}(t)=\operatorname{atan}(\operatorname{Im}(z(t) / \operatorname{Re}(z(t))$. This computation leads to a wrapped phase, which needs to be unwrapped to obtain the linear evolution of the instantaneous phase $\Phi_{u}(t)$, which can be expressed as:

$$
\Phi_{u}(t)=2 \pi f_{b} t+\varphi(t)
$$

where the first term corresponds to the linear phase of the square-wave signal with a beat frequency $f_{b}$ and the second term corresponds to the phase fluctuation $\varphi(t)$ of the modulated RF signal.

Finally subtracting the linear phase and using the expression of the fundamental beat frequency, an estimation $\varphi_{\text {extracted }}(t)$ of the RF signal phase fluctuation is given by:

$$
\varphi_{\text {extracted }}(t)=\Phi_{u}(t)-2 \pi\left|2 f_{s}-f_{c}\right| t
$$

Fig.7 illustrates the result of the phase fluctuation extraction process on a simulated example. The RF signal is generated by an ideal OQPSK modulator with half-sine pulse shaping using a carrier frequency $f_{c}=2.48 \mathrm{GHz}$ and a nominal amplitude of $1 \mathrm{~V}$. The digital ATE channel is modeled by a level-crossing operation (threshold set at $70 \%$ the nominal signal amplitude) followed by a sampling operation with a frequency $f_{s}=$ 
$1.25 \mathrm{GHz}$. The resulting signal is a square-wave signal with a fundamental beat frequency $f_{b}=20 \mathrm{MHz}$. This signal is used as the input of the phase fluctuation extraction process. Results show a very good agreement between the original phase of the RF modulated-signal and the extracted one, with a difference that remains below $2^{\circ}$ and an RMS error of only $0.95^{\circ}$.
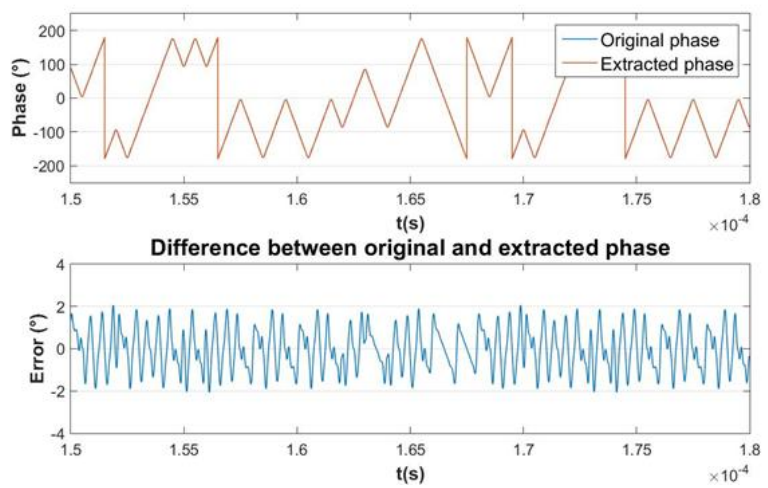

Fig. 7. Comparison between original and extracted phase for a simulated perfect RF modulated-signal.

Note that the expression of the RF signal phase fluctuation in (5) has been established under ideal conditions, i.e. assuming that the carrier frequency is exactly known and that there is a perfect synchronization between the ATE and the DUT. Such conditions are impossible to achieve in a practical environment. In particular, the actual carrier frequency might slightly differ from the ideal one, the synchronization between the ATE sampling frequency and the signal carrier frequency might not be perfect (propagation delay between the DUT and the ATE), or even not possible. These imperfections are actually present in the extracted phase fluctuation, which can be expressed with:

$$
\varphi_{\text {extracted }}(t)=\varphi(t)+2 \pi f_{\text {off }} t+\varphi_{0}
$$

where $\varphi(t)$ is the actual phase fluctuation of the signal under test, $f_{\text {off }}$ is the frequency difference between the assumed carrier frequency and the actual one, and $\varphi_{0}$ is an unknown phase offset related to imperfect or absent synchronization.

These imperfections affect the location of measured points in the constellation. In particular, phase offset causes a rotation of the constellation while the frequency offset induces a spread of the data on the complete trigonometric circle. To ensure accurate EVM measurement, it is clear that both these offsets need to be corrected. While phase offset can be easily identified and removed, frequency offset is more challenging. These corrections will be performed in the second step of the processing algorithm.

\section{2) Amplitude fluctuation extraction}

In parallel of phase fluctuation extraction, a second task is to retrieve the low-frequency amplitude variations that might affect the RF signal envelope. Theoretically an OQPSKmodulated signal with half sine pulse shaping exhibits a constant envelope. However, imperfections in the modulator such as IQ imbalance or power drop can lead to low-frequency amplitude variations. Such variations must be taken into account to obtain accurate EVM measurement.
The implemented solution utilizes the work developed in [12] for power measurement using digital test resources. The fundamental basis comes from the principle of level-crossing that converts voltage-domain information into timing information. This principle is illustrated in Fig.8 in case of an ideal sine-wave that is converted into a square-wave by levelcrossing (with a comparator threshold $C$ different than 0 ). There is a direct relationship between the sine-wave amplitude $A$ and the square-wave duty cycle $\Delta T / T$, and the sine-wave amplitude can be obtained by computing:

$$
A=\frac{C}{\cos \left(\pi \frac{\Delta T}{T}\right)}
$$

where $\Delta T$ is duration between the rising and falling transitions of the square-wave signal, and $T$ is the period of both the sinewave and square-wave signals.

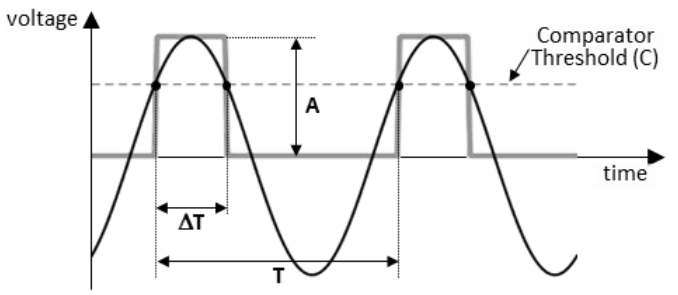

Fig. 8. Conversion from voltage-domain to time-domain through levelcrossing.

Absolute measurement of the signal amplitude using such approach would obviously require a well-controlled comparator threshold. ATE digital channels specify a $\pm 1 \mathrm{mV}$ maximum error on comparison levels. Considering that EVM test is performed at the maximum output power of $+10 \mathrm{dBm}$ (i.e. $1 \mathrm{~V}$ over $50 \Omega$ load) the comparator threshold uncertainty is within $0.1 \%$ of the signal amplitude, which is already very good. In addition, it is worth noting that EVM calculation involves amplitude normalization that only retains envelope fluctuations. Precise absolute amplitude measurement is therefore not required, and the proposed technique can even accommodate few dynamic imperfections in the comparator stage.

According to (6), the sensitivity of the amplitude A to $\square \mathrm{T}$ gets better with increasing values of the comparison threshold C. Yet, one should prevent from any fluctuating amplitude falling below the chosen threshold. With this in mind, we choose an empirical level of 0.7A for the setting of $\mathrm{C}$, which represent a good compromise.

In our context, this principle can be exploited to estimate low-frequency amplitude variations of the RF signal by monitoring the duty cycle of the digital signal captured by the ATE. Indeed, this digital signal corresponds to a square-ware signal generated by a level-crossing operation combined with an under-sampling process. The under-sampling process translates the square-wave signal towards a lower frequency but preserves its essential characteristics, in a limited bandwidth. The variations of the digital signal duty cycle therefore give an image of the variations of the RF signal envelope. 


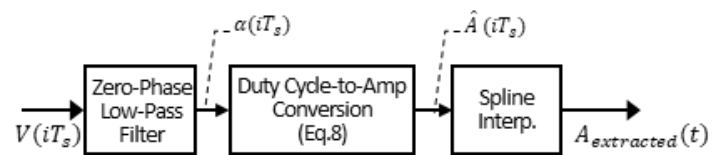

Fig. 9. Amplitude fluctuation extraction from binary vector captured by the ATE.

Practically, the implemented solution involves three consecutive operations, as illustrated in Fig.9. The first one consists in measuring the duty cycle $\alpha=\Delta T / T$ by applying a low-pass filter on the captured digital signal $V\left(i T_{s}\right)$. In this work, we have chosen a type II Chebyshev filter with stopband starting at $7 \mathrm{MHz}$. The filter has been designed to pass the components related to low-frequency envelope variations (i.e. with a bandwidth greater than the chip rate of $2 \mathrm{MHz}$ ), but eliminate all components related to the square waveform of the digital signal (i.e. with a cut-off frequency lower than the fundamental beat frequency of $20 \mathrm{MHz}$ ). Here again, forward-backward filtering is used to implement a zerophase filter. The output of the filter is a discrete-time signal $\alpha\left(i T_{s}\right)$ whose value is representative of the digital signal duty cycle. Duty cycle-to-amplitude conversion is then simply realized based on (6):

$$
\hat{A}\left(i T_{s}\right)=\frac{C}{\cos \left(\pi \alpha\left(i T_{S}\right)\right)}
$$

Finally, spline interpolation is applied to obtain a pseudo continuous-time signal $A_{\text {extracted }}(t)$ that matches with the time resolution of the extracted phase. This signal corresponds to an estimation of the RF signal amplitude fluctuation.
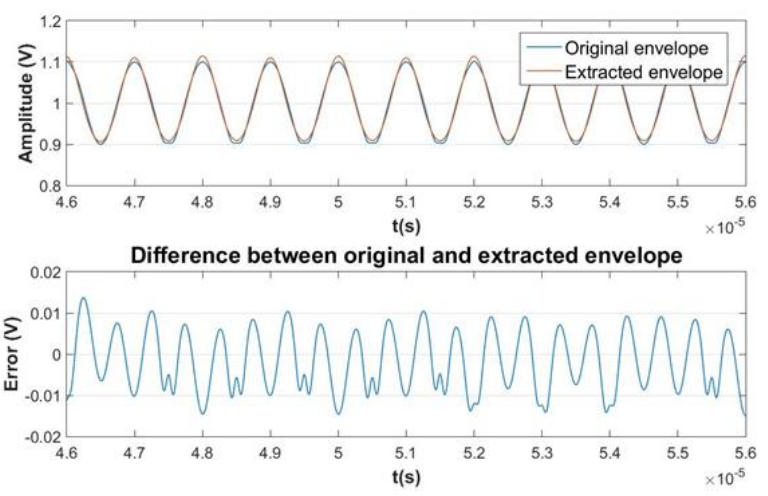

Fig. 10. Comparison between original and extracted envelope for a simulated RF modulated-signal with 10\% IQ imbalance.

Fig.10 illustrates the result of the amplitude fluctuation extraction process on a simulated example. For this simulation, $10 \%$ IQ imbalance has been injected in the OQPSK modulator model in order to generate a RF modulated-signal that presents low-frequency amplitude variations. Results show a very good agreement between the original envelope of the RF signal with a nominal amplitude of $1 \mathrm{~V}$ and the extracted one. The reconstructed envelope is well-centered on the original one and the difference between both curves does not exceed $15 \mathrm{mV}$, with an RMS error of only $6 \mathrm{mV}$. This corresponds to a maximal error below $1.5 \%$ and an RMS error of $0.6 \%$.

\section{B. Step 2: Measurement data preparation and reference data generation}

The second step of the algorithm is dedicated to the preparation of measurement data and the generation of reference data. These operations are realized in parallel, but with some interactions regarding correction of the extracted phase fluctuation and timing alignment of measured and reference data.

\section{1) Reference data generation}

The first task of the reference data generation process is to recover the symbols bits that have been delivered by the DUT. Theoretically, this could be accomplished by a simple sampling of the extracted phase fluctuation at the rate of $2 \mathrm{Mbit} / \mathrm{s}$, where each value of the sampled phase is directly translated into the corresponding symbol bits. However in practice, such a simple process is totally inefficient because of the inevitable phase and frequency offsets present in the signal captured by the ATE. These offsets, even small, affect the extracted phase fluctuation and entail many errors in the decoded data. It is therefore mandatory to perform carrier frequency and phase recovery to ensure a correct detection of the symbol bits.

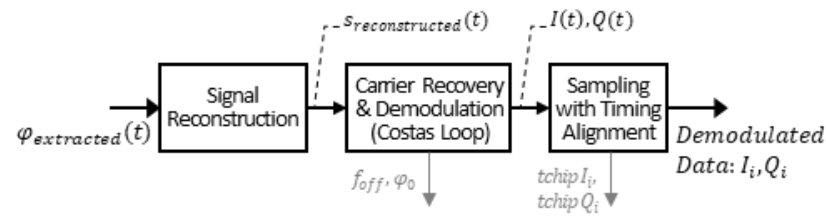

Fig. 11. Detection of symbol bits from extracted phase fluctuation.

The solution that has been implemented relies on a threestep process as illustrated in Fig.11. The first step consists of performing the reconstruction of the RF modulated signal emitted by the DUT by adding the extracted phase fluctuation to the ideal instantaneous linear phase of the carrier:

$$
s_{\text {reconstructed }}(t)=\sin \left(2 \pi f_{c} t \pm \varphi_{\text {extracted }}(t)\right)
$$

Note that depending whether the sampling frequency is chosen with a positive or negative deviation with respect to the carrier half-frequency, the extracted phase fluctuation should be added or subtracted to take into account spectral inversion.

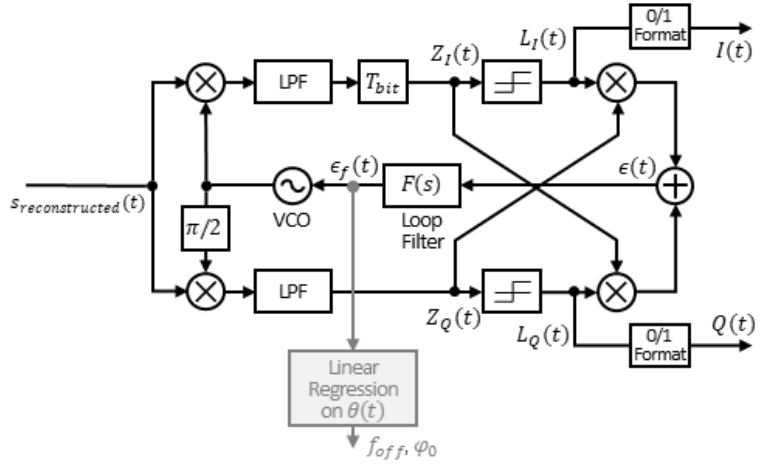

Fig. 12. Costas loop for demodulation of OQPSK signals with pulse shaping.

The second step is dedicated to carrier recovery as well as demodulation. This is accomplished through the use of a Costas loop. A Costas loop is a PLL-based circuit that uses coherent quadrature signals to measure a phase error, which in turn is used to regulate the loop oscillator. When locked, quadrature signals correspond to the demodulated data. In this work, we use a software model of the Costas loop based on the 
design proposed in [13], with modifications to manage OQPSK with half sine pulse shaping. The architecture of the loop is given in Fig.12. The input of the loop is the reconstructed RF signal, which is split in two $I$ and $Q$ branches. Signals on the $I$ and $Q$ branches are respectively multiplied by a VCO-generated tone and a $90^{\circ}$ shifted version of this tone; low pass filtering is then applied to remove the double frequency component and perform the demodulation. By taking the difference between the absolute value of the demodulated $I$ branch and $Q$ branch, an error is obtained; this error $\varepsilon(t)$ passes through a loop filter and drives the VCO to achieve phase and frequency locking. Note that in the specific context of OQPSK with half sine pulse shaping, a delay of one-bit period $\left(T_{b i t}\right)$ is added on the $I$ branch to compensate for the delay that has been added on the $Q$ branch during the modulation process. Finally in the locked state, $Z_{I}(t)$ and $Z_{Q}(t)$ correspond to reconstructed waveforms of the original quadrature signals generated within the DUT and $I(t)$ and $Q(t)$ are the binary representation of these signals.

To improve the locking of the Costas loop, the VCO initial frequency should be set close to the actual carrier frequency of the incoming signal. To do so, a first estimation of the carrier frequency $f_{c_{\text {coarse }}}$ is realized based on the spectrum of the squared RF reconstructed signal, by computing the mean of the two main components located at $2 f_{c} \pm f_{\text {bit }} / 2$ (where $f_{\text {bit }}$ is the $2 \mathrm{MHz}$ bit frequency) and dividing this value by 2 . The accuracy of the estimation depends on FFT resolution but is typically in the range of few $\mathrm{kHz}$. This estimation is not precise enough to perform correct demodulation but constitutes a useful data to set the VCO initial frequency.

Another interest of the Costas loop is that the frequency and phase offsets present in the signal captured by the ATE can be evaluated from a simple processing of its error signal $\varepsilon(t)$. More precisely, the phase correction $\Theta(t)$ performed by the loop can be computed by integrating the filtered error signal $\varepsilon_{f}(t)$ with:

$$
\Theta(t)=\int_{0}^{t} 2 \pi \varepsilon_{f}(t) \cdot d t
$$

As illustrated in Fig.13, this signal exhibits a linear trend that can be fitted with a function of the form $a * t+b$. The slope coefficient depends on the difference between the actual carrier frequency and the initial VCO frequency with $a=$ $2 \pi\left(f_{c_{\text {meas }}}-f_{c_{\text {coarse }}}\right)$, while the intercept term directly corresponds to the initial phase offset $\varphi_{0}$. Consequently, we can estimate the frequency and phase offsets present in the signal captured by the ATE with:

$$
\begin{aligned}
& f_{o f f}=f_{c}-\left(f_{c_{\text {coarse }}}+\frac{a}{2 \pi}\right) \\
& \varphi_{0}=b
\end{aligned}
$$

where $f_{c_{\text {meas }}}$ is the measured value of the actual carrier frequency.

These values will the used to correct the extracted phase fluctuation $\varphi_{\text {extracted }}(t)$ in the branch dedicated to measurement data preparation.

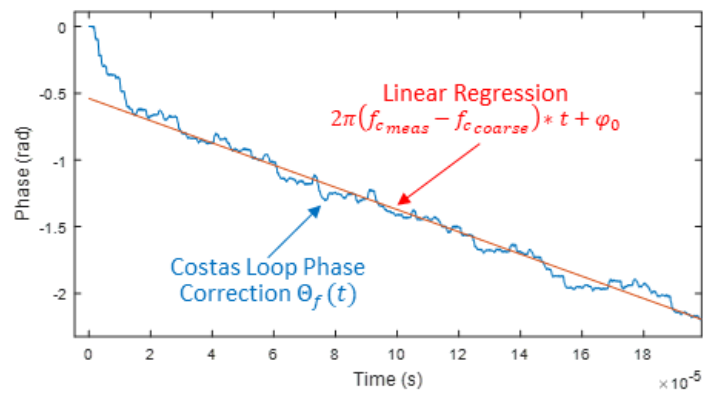

Fig. 13. Linear regression on Costas loop phase correction $\Theta(t)$.

Finally, the last step of symbol bits detection simply consists in sampling the reconstructed $I(t)$ and $Q(t)$ binary signals with a $1 / 2 T_{b i t}$ clock frequency, as illustrated in Fig.14. Note that to ensure correct interpretation, the sampling should occur in the middle of the period of each binary signal, which means that a specific timing alignment has to be defined for each binary signal. Practically to determine the proper alignment, we assume a sampling clock with an initial delay $t_{\text {delay }}=0$. Then for each binary signal, we compute the time difference $\delta t_{I / Q}$ between every signal transition and the following edge of the sampling clock. The mean of these differences over the complete sequence $\left(\overline{\delta t_{I / Q}}\right)$ corresponds to the initial delay that must be applied on the sampling clock to align the sampling clock with the transitions of corresponding binary signal. An additional delay of $T_{b i t}$ is then simply added to sample the data in the middle of the binary signal period:

$$
\text { tdelay }_{I / Q}=\left(\overline{\delta t_{I / Q}}+T_{b i t}\right)
$$

By the end of this process, we therefore have the demodulated data $I$ and $Q$ together with their sampling instants tchipI and tchipQ. These instants will be used later in the algorithm to sample both the measured and reference data.
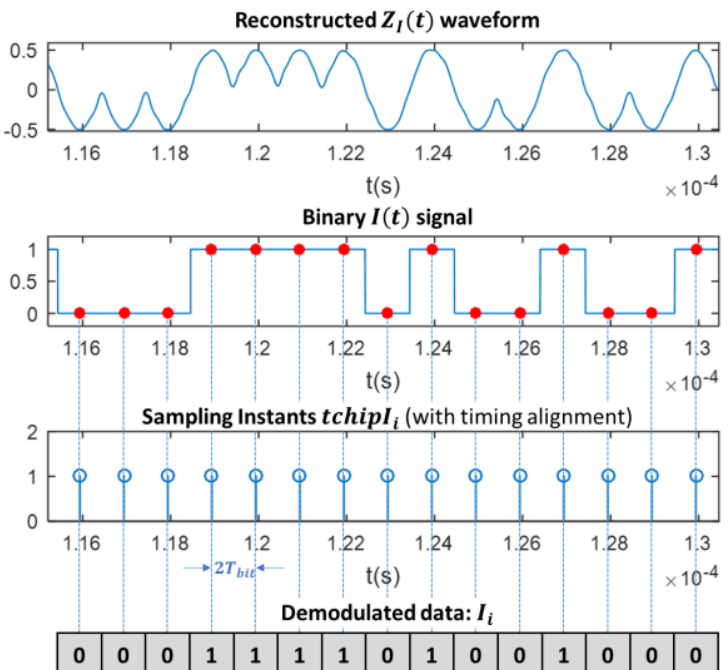

Fig. 14. Example of sampling process on reconstructed $I(t)$ signal.

The following task is to generate a RF reference signal $s_{r e f}(t)$ using the demodulated data. For this, the demodulated data are fed into an ideal modulator model centered on the measured carrier frequency $f_{c_{\text {meas }}}$. The output of the modulator is then filtered by a band-pass filter to consider the 
limited bandwidth of the measurement instrument and synchronized with the reconstructed RF signal. Synchronization is achieved by computing the crosscorrelation between both signals; the maximum value of the cross-correlation function corresponds to the delay that has to be applied to ensure the best timing alignment. For a better accuracy, cross-correlation is not computed on the complete sequence but on limited portions of the sequence and averaging is used. In this work, we use five different portions of the sequence composed of 20 symbol bits.

Finally, the last task is to determine the polar coordinates of the reference data. The radial coordinate is constant whatever the emitted symbol since it is a specific feature of OQPSK with half-sine pulse shaping to have a constant modulation envelope. Its value depends on the amplitude level of the signal emitted by the DUT. It is computed by taking the maximum value of the extracted RF signal amplitude fluctuation:

$$
A_{\text {ref }}=\max \left[A_{\text {extracted }}(t)\right]
$$

The angular coordinate corresponds to the phase of the reference signal $s_{\text {ref }}(t)$; it is computed by taking the angle of the complex representation obtained from Hilbert transform. This reference phase $\varphi_{\text {ref }}(t)$ is then simply sampled at the tchipI and tchipQ instants determined in the first task.

\section{2) Measurement data preparation}

The main task of the measurement data preparation is to correct the extracted phase fluctuation $\varphi_{\text {extracted }}(t)$, taking into account the frequency offset $f_{\text {off }}$ and the phase offset $\varphi_{0}$ determined by the Costas loop in the reference data generation process. This is done by subtracting the supplementary linear phase evolution introduced by the frequency offset and the measure phase offset to the original extracted phase fluctuation:

$$
\varphi_{\text {meas }}(t)=\varphi_{\text {extracted }}(t)-2 \pi f_{\text {off }} t-\varphi_{0}
$$

Finally, this corrected phase fluctuation is then simply sampled at the tchipI and tchipQ instants, as well the extracted amplitude fluctuation to produce the measurement data.

\section{Step 3: EVM calculation}

This last step finalizes the process by implementing the EVM calculation. It takes both the measured and reference data as inputs. Note that in case of OQPSK modulation format, the computation uses two points-per-symbols due to the one-bit period offset between $I$ and $Q$ data. In this case, the computed value is called Offset Error Vector Magnitude (OEVM).

Practically for each decoded symbol, the errors $\delta I_{i}$ and $\delta Q_{i}$ are computed at the $\operatorname{tchip}_{i}$ and $\operatorname{tchip} Q_{i}$ instants with:

$$
\begin{aligned}
\delta I_{i}= & A_{\text {meas }}\left(\operatorname{tchip}_{i}\right) * \cos \left(\varphi_{\text {meas }}\left(\text { tchip }_{i}\right)\right) \\
& -A_{\text {ref }} * \cos \left(\varphi_{\text {ref }}\left(\text { tchip }_{i}\right)\right) \\
\delta Q_{i}= & A_{\text {meas }}\left(\text { tchip }_{i}\right) * \sin \left(\varphi_{\text {meas }}\left(\text { tchip }_{i}\right)\right) \\
& -A_{\text {ref }} * \sin \left(\varphi_{\text {ref }}\left(\text { tchip }_{i}\right)\right)
\end{aligned}
$$

OEVM is then computed as the root mean square of these errors and expressed as a percentage of the reference signal amplitude:

$$
\operatorname{OEVM}(\%)=\frac{\sqrt{\frac{1}{n} \sum_{i=1}^{n}\left(\delta I_{i}{ }^{2}+\delta Q_{i}{ }^{2}\right)}}{A_{\text {ref }}} * 100
$$

\section{VALIDATION}

The software post-processing algorithm has been implemented in Matlab/Simulink®. Hardware measurements have been performed to validate the proposed solution, first with a lab experimental set up and then on an industrial ATE. Our validation approach is based on the comparison between the EVM results obtained by our technique and a reference measure obtained using dedicated RF instrument as currently done in the industrial context. Both absolute EVM and measure variability are addressed in the following.

\section{A. Lab experiments}

\section{1) Setup}

The experimental setup depicted in Fig.15 has been developed to evaluate the proposed solution. In a first version, a Universal Software Defined Radio Peripheral (USRP 2901 from National Instrument $\left.{ }^{\circledR}\right)$ is used to emulate the Device Under Test (DUT). It is programmed to deliver a RF signal with a carrier frequency $f_{c}=2.48 \mathrm{GHz}$ (channel 26 as stated in IEEE Std 802.15.4 ${ }^{\mathrm{TM}}$ ), using OQPSK modulation format with half-sine pulse shaping. The modulator model is implemented with help of GNURadio software. A key interest in using a USRP is that it is possible to add controlled imperfections in the modulator model such as IQ imbalance or phase noise that degrade the quality of the generated signal and therefore allows the evaluation of the proposed solution over a relatively large range of EVM values. On the other hand, because of its versatility, such product is obviously less performing than a dedicated IC and does not permit to generate a signal with EVM values below 1\%. The USRP is therefore replaced by a NXP ZigBee transceiver in a second version, which presents a typical EVM value less than $1 \%$. This IC is also programmed to deliver a RF modulated signal with a carrier frequency $f_{c}=2.48 \mathrm{GHz}$.

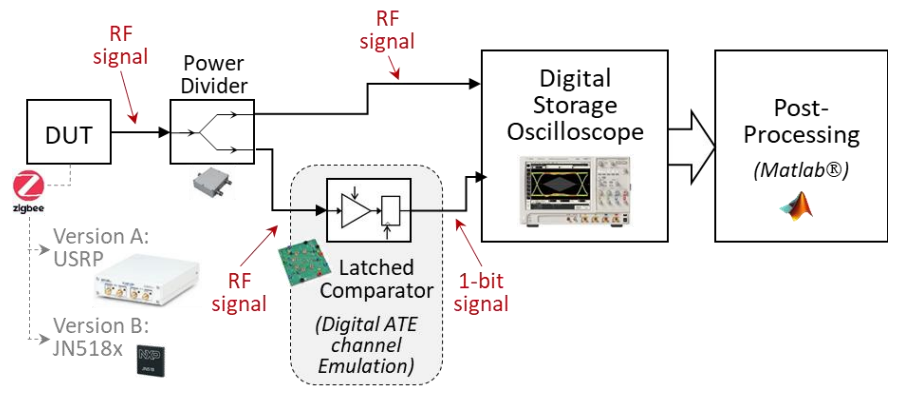

Fig. 15. Experimental setup for lab experiments.

The generated RF signal is split in two signals using a resistive power divider. The first one is directly connected to a fast digital storage oscilloscope and its acquisition is performed at 5 GSamples/s, which satisfies the Nyquist rate. The second one is first sent to a latched comparator (ADCMP 572 from Analog Devices) that emulates the digital tester channel by converting the RF modulated signal into a square-wave signal. 
The comparator threshold is set at $70 \%$ of the nominal $\mathrm{RF}$ signal amplitude and the sampling frequency at $f_{s}=$ $1.25 \mathrm{GHz}$; the output of the latched comparator is therefore a binary signal with a fundamental beat frequency $f_{b}=$ $20 \mathrm{MHz}$. This output is connected to the second channel of the oscilloscope, which performs its acquisition. Both signals captured by the oscilloscope are then transferred on a PC for further processing. The complete oscilloscope acquisition at 5 GSamples/s is conserved for the analysis of the RF signal, while decimation by a factor 4 is applied on the binary signal to keep only $1.25 \mathrm{GSamples} / \mathrm{s}$ in order to match with the latched comparator sampling frequency. Note that this setup does not provide the best experimental conditions because there is no synchronization between the sampling clock of the latched comparator and the oscilloscope. Finally, EVM measurement algorithm is applied on the original RF signal and on the one reconstructed from the binary acquisition.

2) Results with DUT emulation (NI USRP)

First experiments have been performed using the USRP as DUT emulation. Initial measurements have been realized on the signal generated by the USRP without adding any impairment, which gives the floor OEVM of the device. Then additional measurements have been realized under degraded conditions, i.e. with different levels of phase noise injected on the reference VCO of the modulator and with an IQ imbalance of 5\%. Results are summarized in Fig.16 and Table II.

Analyzing these results, we can observe a good correlation between the values measured on the RF signal and the ones computed from the 1-bit capture, on the range between $1 \%$ to $7 \%$. The difference between those values is inferior to $0.1 \%$ for EVM below 4\% and slightly increases for higher EVM. The maximum difference is observed in case of added IQ imbalance, possibly because of the amplitude envelope reconstruction. Yet, the error remains lower than $0.5 \%$ for a measured EVM around 6\%. Overall, the average error is only of $-0.03 \%$ and the RMS error of $0.19 \%$. These results clearly demonstrate the potential of the proposed solution to perform EVM measurements based only on a digital acquisition.

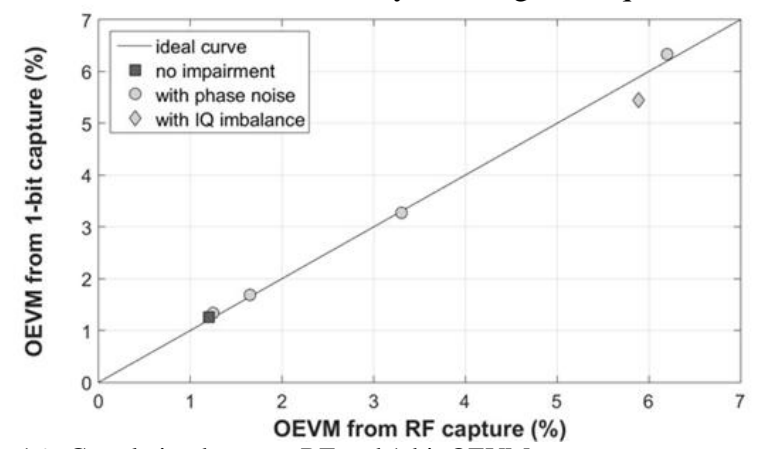

Fig. 16. Correlation between RF and 1-bit OEVM measurements.

TABLE II.

COMPARISON BETWEEN OEVM MEASUREMENTS FROM RF AND 1-BIT ACQUISITIONS ON USRP DEVICE

\begin{tabular}{|l|l|l|l|}
\cline { 2 - 4 } \multicolumn{1}{c|}{} & RF capture & 1-bit capture & Difference \\
\hline \hline no impairment & $1.20 \%$ & $1.25 \%$ & $+0.05 \%$ \\
\hline \hline \multirow{3}{*}{ with phase noise } & $1.25 \%$ & $1.34 \%$ & $+0.09 \%$ \\
\cline { 2 - 4 } & $1.65 \%$ & $1.68 \%$ & $+0.03 \%$ \\
\cline { 2 - 4 } & $3.30 \%$ & $3.27 \%$ & $-0.03 \%$ \\
\hline
\end{tabular}

\begin{tabular}{|l|l|l|l|}
\hline & $6.20 \%$ & $6.34 \%$ & $+0.14 \%$ \\
\hline \hline with IQ imbalance & $5.89 \%$ & $5.45 \%$ & $-0.44 \%$ \\
\hline \hline
\end{tabular}

3) Results on actual IC (NXP JN518x ZigBee transceiver)

Further experiments have been performed replacing the USRP by a real ZigBee transceiver from NXP Semiconductors. Under nominal setting, this device delivers an RF modulated-signal with a better OEVM than the USRP, i.e. well below $1 \%$. The quality of the signal can be slightly degraded through the programing of an internal configuration register. There are two possible degraded settings that correspond to a voluntarily wrong programming of the device frequency synthesizer registers, likely increasing the phase noise.

For each setting, the parallel acquisition of the RF signal and the binary signal delivered by the latched comparator has been realized and OEVM values have been determined. Moreover, in order to study the repeatability of the measurement, this process has been repeated 50 times. Results are illustrated in Fig.17 which shows the histograms of measured OEVM values and measurement statistics are summarized in Table III. A very good agreement can be observed between measurements realized on the RF signal and the ones derived from the 1-bit signal. The difference between the mean OEVM value determined on the RF signal and the one computed on the 1-bit signal does not exceed $0.04 \%$ over the three different settings, clearly demonstrating the ability of the proposed digital solution to perform accurate EVM measurements. Both solutions also lead to a similar dispersion, indicating that there is no degradation of the measurement repeatability by exploiting the binary signal.

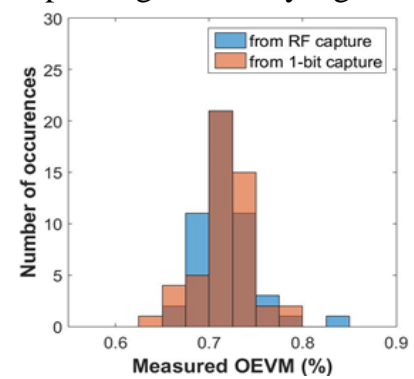

(a) Nominal setting

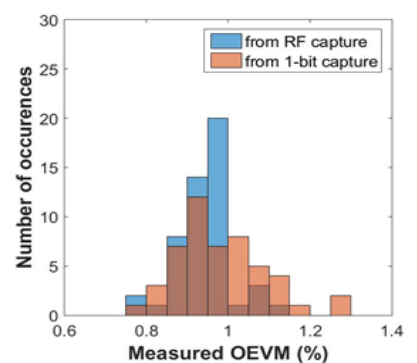

(b) Degraded setting \#1

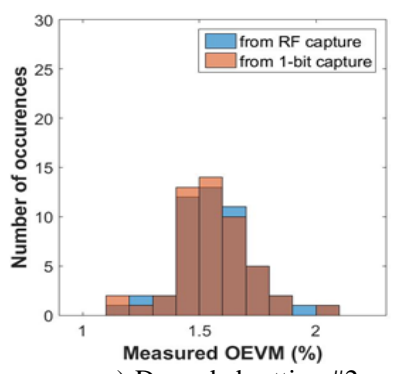

c) Degraded setting \#2
Fig. 17. Histograms of measured OEVM values with lab experimental setup for different settings of JN518x IC.

TABLE III.

STATISTICS OF OEVM MEASUREMENTS FROM RF AND 1-BIT ACQUISITIONS ON JN518X IC WITH LAB EXPERIMENTAL SETUP

\begin{tabular}{|l|l|l|l|}
\hline \hline \multirow{2}{*}{ Nominal setting } & Mean & $0.72 \%$ & $0.72 \%$ \\
\cline { 2 - 4 } & Std Dev & $0.03 \%$ & $0.03 \%$ \\
\hline \hline
\end{tabular}




\begin{tabular}{|l|l|l|l|}
\hline \hline \multirow{2}{*}{ Degraded setting \#1 } & Mean & $0.94 \%$ & $0.98 \%$ \\
\cline { 2 - 4 } & Std Dev & $0.07 \%$ & $0.11 \%$ \\
\hline \hline \multirow{2}{*}{ Degraded setting \#2 } & Mean & $1.57 \%$ & $1.56 \%$ \\
\cline { 2 - 4 } & Std Dev & $0.16 \%$ & $0.16 \%$ \\
\hline
\end{tabular}

\section{B. Validation on industrial setup}

Finally, to corroborate the results obtained with the lab experimental setup, the technique has been implemented on an industrial Advantest V93k ATE, as depicted Fig.18. A JN518x device is first measured using the conventional industrial setup, i.e. with an acquisition realized by an RF tester channel (PortScale RF®) and using the built-in ATE processing functions. Then, the device is connected to a standard digital tester channel (PinScale 1600®) and a second acquisition is realized (device carrier frequency set at $f_{c}=2.405 \mathrm{GHz}$ and ATE sampling frequency set at $f_{s}=1.212 \mathrm{GHz}$, which results in a baseband digital signal with a fundamental beat frequency $f_{b}=19.2 \mathrm{MHz}$ ). Binary data captured by the digital tester channel are transferred to a PC and processed by the dedicated post-processing algorithm to evaluate the EVM performance. Note that, contrarily to the lab experiments, the RF and digital acquisitions are not realized in parallel but in a sequential manner because the ATE load board was not designed to allow simultaneous connection of the device to both RF and digital tester channels. Also note that, for logistical reasons (different locations of ATE and lab facilities), the device measured in this experiment is not the same than the one measured in the previous section and is mounted on a different test board.

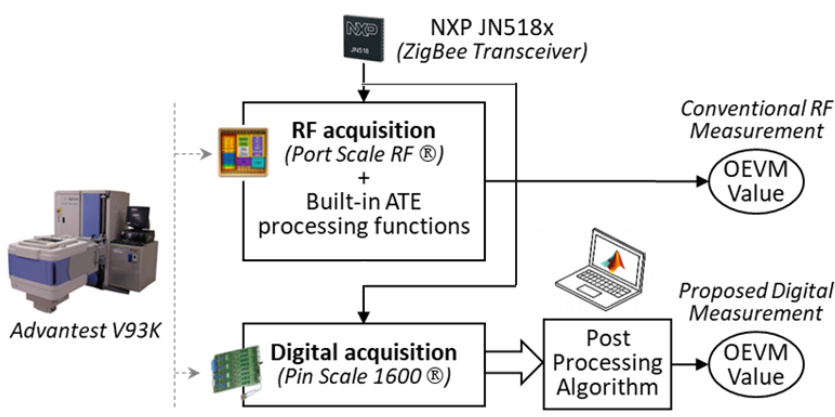

Fig. 18. Synopsis of validation on industrial ATE.

As for the lab experiments, measurements have been performed under the three different settings of the JN518x device; the RF and digital acquisitions have also been repeated 50 times for each setting in order to study the measurement repeatability. Results are reported in Fig.19 which shows the histograms of measured OEVM values using the conventional and the proposed digital methods. Measurement statistics are summarized in Table IV, in terms of mean value and standard deviation achieved for each setting.

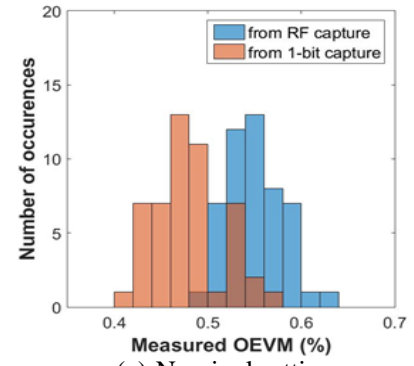

(a) Nominal setting
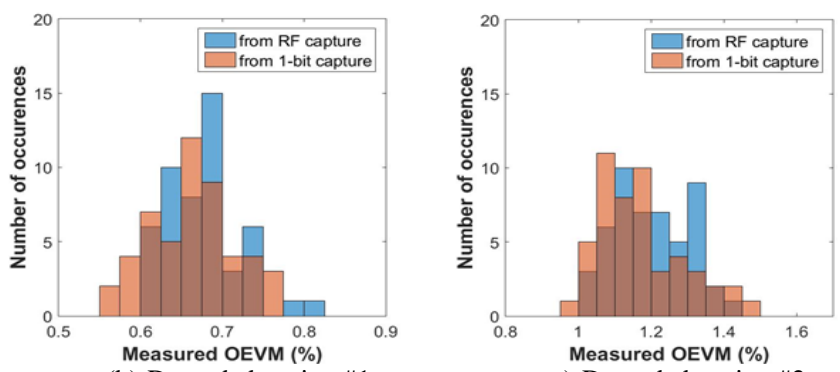

(b) Degraded setting \#1

c) Degraded setting \#2

Fig. 19. Histograms of measured OEVM values with industrial ATE setup for different settings of JN518x IC.

Globally, these results confirm the good agreement between the conventional RF measurement and the implemented digital solution. Indeed, the difference in the mean OEVM value determined from the RF acquisition and the one computed from the digital capture remains significantly below $0.1 \%$, with a difference of $0.07 \%$ for the nominal setting, $0.02 \%$ for degraded setting \#1 and $0.03 \%$ for degraded setting \#2. Note that the difference of $0.07 \%$ observed in the nominal setting is not really meaningful because of the extremely low EVM level, knowing that the typical EVM floor of up-to-date RF measurement instrumentation is between 0.5 and $1 \%$. Regarding dispersion, the standard deviation computed over the 50 runs in each setting is similar when using the conventional RF measurement or the digital one, confirming that the digital solution does not introduce a degradation of the measurement repeatability.

TABLE IV.

STATISTICS OF OEVM MEASUREMENTS FROM RF AND 1-BIT ACQUISTIONS ON JN518X IC WITH INDUSTRIAL ATE SETUP

\begin{tabular}{|l|l|l|l|}
\cline { 3 - 4 } \multicolumn{2}{c|}{} & RF capture & 1-bit capture \\
\hline \hline \multirow{2}{*}{ Nominal setting } & Mean & $0.55 \%$ & $0.48 \%$ \\
\cline { 2 - 4 } & Std Dev & $0.03 \%$ & $0.03 \%$ \\
\hline \hline \multirow{2}{*}{ Degraded setting \#1 } & Mean & $0.68 \%$ & $0.66 \%$ \\
\cline { 2 - 4 } & Std Dev & $0.04 \%$ & $0.05 \%$ \\
\hline \hline \multirow{2}{*}{ Degraded setting \#2 2} & Mean & $1.20 \%$ & $1.17 \%$ \\
\cline { 2 - 4 } & Std Dev & $0.10 \%$ & $0.12 \%$ \\
\hline \hline
\end{tabular}

These results fully validate the proposed strategy which permits to drastically reduce the cost of the required test equipment while maintaining the ability to perform accurate EVM measurements.

\section{CONCLUSION}

EVM, together with spectral masking and output power is one the major specification that must be verified for an RF transmitter during manufacturing test. So far, this characterization involves high-priced RF instrumentation. In 
this paper, we demonstrated that EVM can be measured from a simple 1-bit direct under-sampling of the RF signal. Results are obtained on a $2.4 \mathrm{GHz}$ Zigbee ${ }^{\circledR}$ transmitter using OQPSK modulation scheme. The actual sampling frequency complies with standard ATE digital resources that have a cut-off frequency of $1.6 \mathrm{GHz}$.

The proposed approach relies on DSP techniques to retrieve signal characteristics from the captured bitstream. In addition, the detailed algorithm does not require synchronization

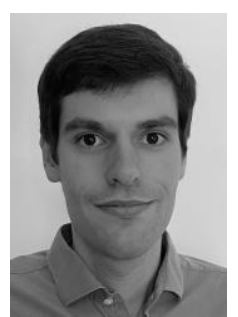
between the RF modulator to be tested and the sampling system. Such approach has already been successfully applied to power and spectral mask testing. Novelty here is the ability to address EVM calculation with extensive experimental results.

Presented results have been obtained in simulation and in various experimental environments including laboratory test bench and industrial test floor. In these experiments, real transmitters have been used together with software-radio RF synthesizers to emulate DUT defects such as noise or IQ imbalance. In all cases, very good matches have been observed between validation approaches. When compared to actual industrial testing involving RF instruments, our method does not produce differences above $0.07 \%$ around a $0.6 \%$ EVM which is within the measure uncertainty for both techniques.

Although OQPSK was the only modulation under concern here, the proposed method should be suitable for similar bandlimited modulation schemes such as FSK or GMSK. If amplitude modulation is involved, such as in QAM, results will probably depend on the expected AM depth because comparator threshold must accommodate the lowest amplitude, leading to a loss of resolution for higher amplitudes.

\section{REFERENCES}

[1] David Mercer, "Global Connected and IoT Device Forecast Update", Strategy Analytics Consumer Electronics, pp. 1-6, May 2019.

[2] F. Demmerle, "Integrated RF-CMOS Transceivers challenge RF Test," Proc. IEEE International Test Conference (ITC), pp. 1-8, 2006.

[3] I. Kore et al., "Multi-site test of RF transceivers on low-cost digital ATE," Proc. IEEE International Test Conference (ITC), pp. 1-10, 2011.

[4] C. H. Peng et al., "A novel RF self test for a combo SoC on digital ATE with multi-site applications," Proc. IEEE International Test Conference (ITC), pp. 1-8, 2014

[5] M. Ishida K. Ichiyama, "An ATE System for Testing RF Digital Communication Devices With QAM Signal Interfaces," IEEE Design \& Test, vol. 33, no. 6, pp. 15-22, 2016.

[6] C.C. Su et al., "Dynamic analog testing via ATE digital test channels", Proc. IEEE Asian Test Symp. (ATS), pp. 308-312, 2004.

[7] N. Pous et al., J.,"A Level-Crossing Approach for the Analysis of RF Modulated Signals using only Digital Test Resources", J. of Electronic Testing: Theory and App. (JETTA), vol. 27, no. 3, pp 289-303, 2011

[8] S. David-Grignot et al., "Low-cost phase noise testing of complex RF ICs using standard digital ATE," Proc. IEEE International Test Conference (ITC), pp. 1-9, 2014.
[9] W. Van Moer, Y. Rolain, "An Improved Broadband Conversion Scheme for the Large-Signal Network Analyzer," IEEE Trans. on Instrumentation and Measurement, vol. 58, no. 2, pp. 483-487, 2009.

[10] C. Nader et al., "Harmonic Sampling and Reconstruction of Wideband Undersampled Waveforms: Breaking the Code," IEEE Trans. on Microwave Theory and Techniques, vol. 59, no. 11, pp. 2961-2969, 2011.

[11] T. Vayssade et al., "Low-cost digital test solution for symbol error detection of RF ZigBee transmitters", IEEE Trans. On Device \& Materials Reliability, vol. 19, no. 1, pp. 16-24, 2019.

[12] T. Vayssade et al., "Power measurement and spectral test of ZigBee transmitters from 1-bit under-sampled acquisition", Proc. IEEE European Test Symposium (ETS), pp. 1-6, 2019

[13] M. Tytgat, M. Steyaert, P. Reynaert, "Time Domain Model for Costas Loop Based QPSK Receiver," PRIME; Conference on Ph.D. Research in Microelectronics \& Electronics, pp. 1-4, 2012

T. Vayssade received the M.S. degree in Electrical Engineering from the University of Montpellier, France, in 2017.

He is currently a Ph.D. student at LIRMM, Montpellier, France, under a CIFRE agreement with NXP Semiconductors.

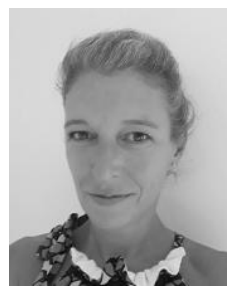
He works on the development of low-cost digital test solutions for RF devices.

F. Azaïs received the M.S. and Ph.D. degrees in Electrical Engineering from the University of Montpellier, France, in 1993 and 1996 respectively.

She is currently a CNRS researcher at LIRMM, Montpellier France. Her main research interests are

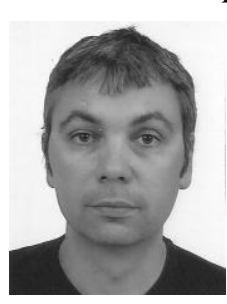
AMS/RF circuit testing, and reliability of integrated systems.

L. Latorre received the M.S. degree in Mechanical and Electronic Engineering from the University of Montpellier, France, in 1995 and the Ph.D. degree in Electrical Engineering from the University of Montpellier, France, in 1999.

He is currently head of the Microelectronics department at LIRMM, Montpellier, France. His main research interests are low-power embedded systems, signal processing, test, and

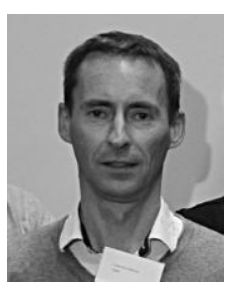
integrated front-end electronics for sensors.

F. Lefèvre received the M.S. degree in Electronic Engineering from the University of Grenoble, France, in 1994.

$\mathrm{He}$ is currently with NXP Semiconductors, Caen, France. He leads the Design for Test definition of large RF, mixed-signal and digital ICs activities. 